\title{
OLEORRESINA DE COPAIBA COMO MATERIA PRIMA PARA LA PRODUCCIÓN DE BIODIÉSEL
}

\author{
Anny Astrid ESPITIA CUBILLOS*, Arnoldo Emilio DELGADO TOBÓN y \\ William Arnulfo APERADOR CHAPARRO
}

Universidad Militar Nueva Granada, Carrera 11, 10180 Bogotá, Colombia

* Autor para correspondencia: anny.espitia@unimilitar.edu.co

(Recibido octubre 2016; aceptado agosto 2017)

Palabras clave: biocombustibles, transesterificación, propiedades térmicas, flujo en frío

\section{RESUMEN}

La investigación consistió en analizar el potencial de la oleorresina extraída del árbol Copaifera reticulata para la producción de biodiésel a partir del proceso de transesterificación. La caracterización incluyó el estudio de la fluidez a bajas temperaturas, la estabilidad oxidativa y la determinación de propiedades como viscosidad, índice de acidez y de fluidez, humedad, y punto de inflamación de la oleorresina tanto cruda como modificada por vía química. Se observó que la técnica empleada para la producción de biodiésel genera pérdidas de oleorresina, ya que el rendimiento global en la producción fue cercano al $10 \%$. Sin embargo, los procesos de refinado y transesterificación influyeron positivamente sobre la estabilidad oxidativa de la oleorresina. Respecto de la oleorresina cruda, la estabilidad de la oleorresina refinada y el biodiésel de copaiba se incrementaron en 46 y $47^{\circ} \mathrm{C}$, respectivamente. De acuerdo con los puntos de fluidez, en todos los casos el comportamiento de flujo en frío supera significativamente los rangos establecidos. El biodiésel presentó un punto de fluidez inferior a $-39^{\circ} \mathrm{C}$. Según los valores de viscosidad y densidad, el uso de la oleorresina en sus formas cruda o modificado - aunque condicionado de forma directa- podría considerarse en mezclas con gasóleo u otros biocombustibles, a fin de mejorar sus propiedades a bajas temperaturas. Estudios posteriores permitirán establecer técnicas eficientes para la modificación química de la oleorresina.

Key words: biofuels, transesterification, thermal properties, biofuels, cold flow

\begin{abstract}
On this research, the potential of oleoresin extracted from the tree Copaifera reticulata was analyzed for the production of biofuel starting from the transesterification process. Properties like viscosity, acid, moisture, flash point and melt fluidity index were determined for both the raw and the chemically modified oleoresin. Its behavior on low temperatures and its oxidative stability were included on the characterization. It was determined that the route used for the production of biodiesel is not suitable because it generates a high loss of oleoresin, since the overall performance in biofuel production was approximately $10 \%$. Still, refining processes and transesterification had a positive effect on the oxidative stability of oleoresin. With respect to raw oleoresin, the
\end{abstract}


stability of refined oleoresin and copaiba biodiesel increased to 46 and $47^{\circ} \mathrm{C}$, respectively. According to the fluidity points, in all cases the behavior on cold fluidity significantly exceeds the established ranges. Copaiba biodiesel showed a fluidity point below $-39^{\circ} \mathrm{C}$. According to the values of viscosity and density, the use of oleoresin in its raw or modified forms, although directly conditioned, could be considered in mixtures with gasoil or other biofuels to improve their low temperature properties. Further studies are needed to establish efficient routes for the chemical modification of oleoresin.

\section{INTRODUCCIÓN}

El aumento anual del parque automotor a escala mundial tiene un impacto importante en el consumo total de energía primaria y la contaminación atmosférica urbana. Lo anterior, unido a la preocupación general por el carácter no renovable de los combustibles fósiles, ha promovido el desarrollo de combustibles alternativos, en especial los de origen renovable (CEPAL 2007, Delgado et al. 2011, Gaurav et al. 2017).

Desde la perspectiva según la cual lo ideal es reducir la emisión de $\mathrm{CO}_{2}$, el uso de aceites extraídos de plantas oleaginosas como combustibles constituye una opción ecológica, dadas las ventajas inherentes a la utilización de biocombustibles, como su carácter biodegradable y atóxico, la reducción de emisiones de $\mathrm{CO}$ y $\mathrm{CO}_{2}$, la ausencia de azufre y su poder lubricante (Agarwal 2007, Demirbas 2007, Mata et al. 2010, Atabani et al. 2012, Sitepu et al. 2014, Habibullah et al. 2015, Morone y Cottoni 2016).

La transesterificación o alcohólisis es la vía más usada para la producción de biodiésel. Durante este proceso, los triglicéridos del aceite vegetal reaccionan con un alcohol de cadena corta, usualmente metanol, en presencia de un catalizador alcalino, para producir alquil ésteres de ácidos grasos (biodiésel) y glicerol como subproducto. El biocombustible que se obtiene con este método es de menor viscosidad con relación al aceite vegetal de partida, lo cual disminuye los problemas relacionados con la inyección de combustible en el motor y su fluidez a bajas temperaturas (Rashid y Anwar 2008, Atabani et al. 2012, Malins et al. 2014, Habibullah et al. 2015, Kuss et al. 2015, Rathore et al. 2016).

El biodiésel se puede producir a partir de cualquier aceite vegetal (virgen o usado) o de grasas animales, mediante el proceso de transesterificación. Incluso nuevas investigaciones informan sobre su generación a partir de microalgas y otras describen el uso de oleorresinas para tal fin. Se dice que debido a la naturaleza de la composición química de la oleorresina extraída del árbol de copaiba, es posible utilizarla directamente como combustible o en mezclas con gasóleo para motores de combustión interna (Calvin 1980, 1983, 1987, Veiga-Junior y Pinto 2002, Chen et al. 2009, Pieri et al. 2009).

La oleorresina de copaiba se obtiene del tronco de árboles pertenecientes a la especie Copaifera officinalis (de la familia Fabaceae, subfamilia Caesalpinioideae), la mayor parte de los cuales se hallan en zonas tropicales de América Latina, particularmente en la región amazónica. Está compuesta principalmente por sesquiterpenos, sobre todo hidrocarburos sesquiterpénicos como $\alpha$-copaeno, trans $\beta$-cariofileno y $\alpha$-humuleno, y diterpenos como los ácidos copaíbico, copálico y copaiférico. Sin embargo, su composición puede variar considerablemente dependiendo de la especie y de los factores medioambientales (Herrero-Jáuregui et al. 2011, Leandro et al. 2012, Gelmini et al. 2013).

En general, la cantidad de oleorresina que se puede extraer de un árbol puede estar sujeta tanto a factores bióticos como abióticos. Algunos autores afirman que aspectos como el diámetro del árbol, la edad, la estación y la infestación de termitas pueden influir en la cantidad de oleorresina que es factible recolectar en cada cosecha (Da Silva Medeiros y Vieira 2008, Herrero-Jáuregui et al. 2011).

De acuerdo con el estudio realizado por Klauberg et al. (2014), en promedio se pueden recolectar $2.1 \mathrm{~L}$ por árbol en cada cosecha, cantidad que disminuiría en el siguiente ciclo dependiendo de la capacidad de regeneración del árbol, relacionada con la actividad fisiológica de los especímenes — mucho mayor en árboles jóvenes - y con su diámetro. Según $\mathrm{Da}$ Silva Medeiros y Vieira (2008), la producción de oleorresina podría ser ecológicamente sostenible para árboles con diámetros de 30 a $41 \mathrm{~cm}$ respecto de árboles de diámetro superior, en relación con el volumen de oleorresina extraído en cada cosecha y en cuanto a su capacidad de regeneración.

La oleorresina de copaiba se utiliza tradicionalmente en el tratamiento de múltiples enfermedades como analgésico, antiinflamatorio, cicatrizante y protector gástrico, entre otros, y en la atención primaria de salud en poblaciones indígenas (Da Silva 
Medeiros y Vieira 2008, Santos et al. 2008, Rodrigues et al. 2011, Gonçalves et al. 2014, Bardají et al. 2016). Sin embargo, su uso en actividades menos convencionales, como el funcionamiento de pequeñas embarcaciones y la iluminación en comunidades indígenas, ha despertado el interés sobre sus propiedades como combustible (Silva et al. 2010).

En el presente trabajo se analizó el potencial de la oleorresina de copaiba para la producción o formulación de biocombustibles. Se utilizó oleorresina extraída de árboles de Copaifera reticulata para la producción de biodiésel y se analizaron las variaciones en las propiedades de flujo en frío, su estabilidad oxidativa y su capacidad calorífica en las etapas del proceso.

\section{MATERIALES Y MÉTODOS}

La oleorresina de copaiba (OC) se obtuvo de incisiones en árboles de Copaifera reticulata en la amazonia brasileña, a una altitud menor de $300 \mathrm{msnm}$ en clima ecuatorial selvático, en junio de 2016. Se utilizó papel de filtro para eliminar sólidos en suspensión (retención de partículas: $6 \mu \mathrm{m}$ ) y sulfato de sodio anhidro de grado analítico para reducir la humedad.

\section{Neutralización}

La oleorresina secada, filtrada y precalentada se mezcló en proporción 2:1 con una disolución de $\mathrm{NaOH}$ al $10 \%$ y a $30^{\circ} \mathrm{C}$ durante $10 \mathrm{~min}$. Terminada la reacción, la temperatura se aumentó a $75^{\circ} \mathrm{C}$ para separar las fases. Finalmente, la oleorresina refinada se lavó con agua desionizada hasta lograr que su $\mathrm{pH}$ fuera neutro. La humedad en el aceite refinado se eliminó con la adición de $\mathrm{Na}_{2} \mathrm{SO}_{4}$ anhidro en proporción 4:1 con relación al aceite. La eficiencia del proceso de neutralización se estimó según su capacidad para reducir la acidez en la oleorresina, mediante la ecuación (1):

$\%$ eficiencia $=\frac{P i-P r}{P i}$

donde $P i$ es la acidez inicial y $P r$ es la acidez de la oleorresina posterior a la etapa de refinado.

\section{Transesterificación}

La oleorresina refinada y precalentada a la temperatura de operación $\left(\mathrm{T}=60^{\circ} \mathrm{C}\right)$ se mezcló con una disolución de alcohol metílico e hidróxido de sodio. Se empleó una relación másica 4:1 oleorresina/metanol y $1.1 \%$ de $\mathrm{NaOH}$ como catalizador. La mezcla de reacción se mantuvo a $60^{\circ} \mathrm{C}$ durante 90 min a 600 rpm. El montaje empleado para la transesterificación se presenta en la figura 1. Finalizado el tiempo de reacción, se depositó la mezcla en un embudo de decantación, separándola en dos fases, a saber: la fase superior rica en metil ésteres, y la fase inferior que contenía la glicerina, el exceso de alcohol, trazas de catalizador e impurezas.

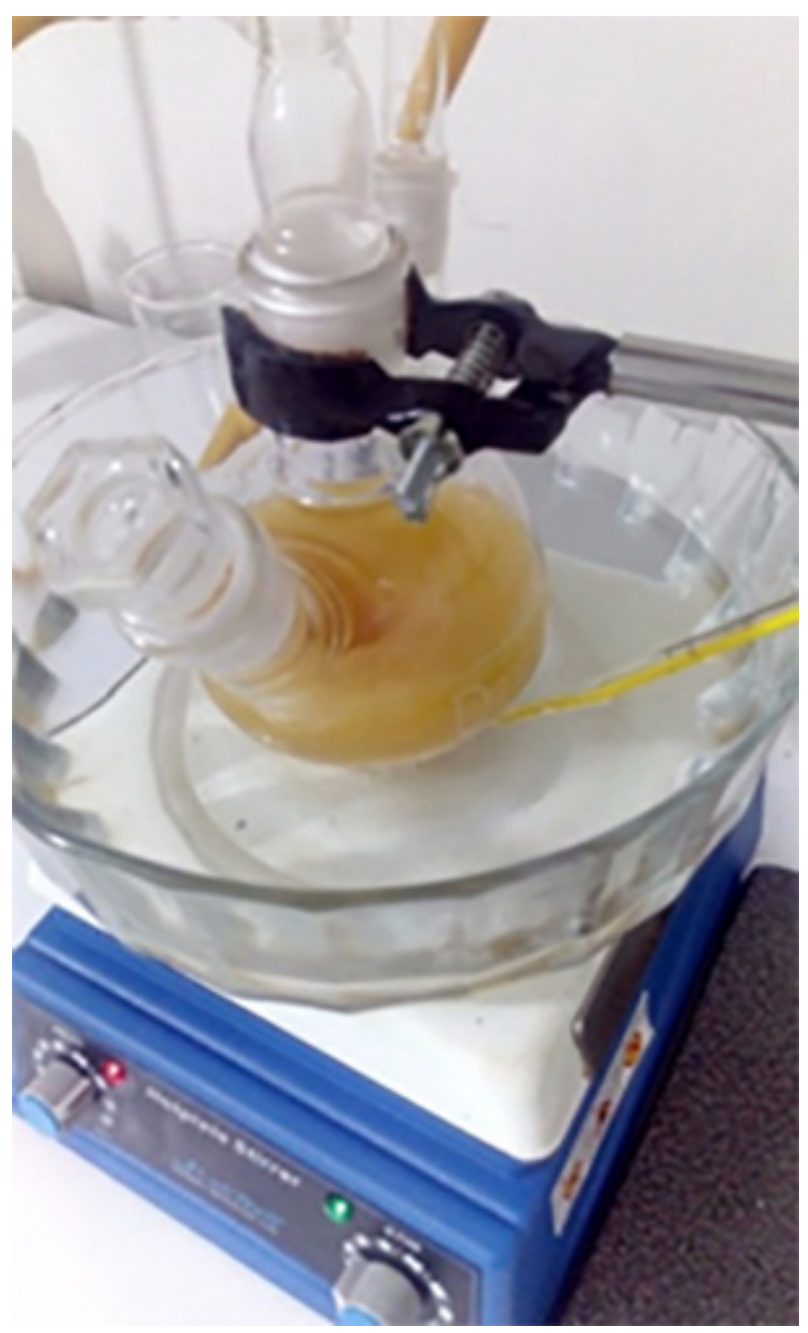

Fig. 1. Montaje experimental: transesterificación

La fase rica en metil ésteres o biodiésel se lavó con agua desionizada seis veces, con una relación 1:1/3 para neutralizar las trazas de catalizador presentes en la mezcla. Finalmente, el agua residual en el biodiésel se eliminó con la adición de $\mathrm{Na}_{2} \mathrm{SO}_{4}$ anhidro. El rendimiento en la producción de biodiésel se calculó con la ecuación (2): 


$$
\text { Rendimiento }=\frac{\begin{array}{c}
g \text { de biodiésel } \\
\text { producido }
\end{array}}{\begin{array}{c}
\text { de muestra tomados } \\
\text { para reacción }
\end{array}} \times 100
$$

Caracterización física y química de la oleorresina Se determinaron el índice de acidez (NTC 218-99 [ICONTEC 1999]), índice de yodo (AOC920 159), índice de saponificación (AOAC 920 160), humedad (ASTM E203 [ASTM 2016a]) y el contenido de fósforo (ET ISO 10540-1-12 [ESA 2012]). El análisis del contenido de ácidos grasos en la oleorresina fue realizado por laboratorios Mol Labs mediante cromatografía de gases (CG) (método FAMES, Mol Labs Ltda.). En la caracterización se determinaron la viscosidad cinemática (ASTM D445 [ASTM 2015a]), el punto de inflamación (ASTM D92 [ASTM 2005a]), la capacidad calorífica (Cp) (ASTM E1269 [ASTM 2005b]) y el punto de fluidez (ASTM D97 [ASTM 2005c), propiedades que se compararon con los valores estándar para el biodiésel, según las normas de la Sociedad Americana para Pruebas y Materiales (ASTM, por sus siglas en inglés) y valores encontrados en la literatura.

\section{Análisis estructural por espectroscopia FTIR}

Se realizó el análisis de la composición estructural de la oleorresina por espectroscopia infrarroja con transformada de Fourier (FTIR), empleando un espectrómetro Nicolet iS10 (Thermo Fisher Scientific), equipado con un sistema de muestreo de reflectancia total atenuada horizontal (HATR). Los espectros FTIR-HATR se obtuvieron con una resolución de $4 \mathrm{~cm}^{-1}$ en el rango espectral de $4000-650 \mathrm{~cm}^{-1}$, con 32 barridos en cada caso. Se empleó el programa OMNIC 9.1.27 (Thermo Fisher Scientific Inc.) para el análisis de los espectros.

En el estudio de las propiedades térmicas de la OC se utilizó un calorímetro diferencial de barrido (DSC, por sus siglas en inglés) serie Q20 (TA Instruments). Para el análisis del comportamiento de la oleorresina a bajas temperaturas, las muestras se purgaron con flujo de $\mathrm{N}_{2}$ de $50 \mathrm{~mL} / \mathrm{min}$. Posteriormente, la muestra se mantuvo en condiciones isotérmicas $\left(50^{\circ} \mathrm{C}\right)$ durante cinco minutos. Transcurrido ese tiempo, el sistema se enfrió a $-60{ }^{\circ} \mathrm{C}$ a una velocidad de $10{ }^{\circ} \mathrm{C} /$ min. Cuando las muestras alcanzaron esta temperatura, se calentó el sistema a $50^{\circ} \mathrm{C}$ a una velocidad de 10 ${ }^{\circ} \mathrm{C} / \mathrm{min}$. Las curvas de enfriamiento y calentamiento obtenidas se emplearon para determinar los puntos de cristalización y fusión, así como las entalpías asociadas a los cambios de fase, cristalización $\left({ }^{\circ} \Delta H_{\mathrm{c}}\right)$ y fusión $\left({ }^{\circ} \Delta H_{\mathrm{m}}\right)$, respectivamente.
Para analizar la degradación termooxidativa de la oleorresina, las muestras se depositaron en un crisol de aluminio abierto y se purgaron con un flujo $50 \mathrm{~mL} /$ min de aire. Posteriormente se calentaron de 10 a 250 ${ }^{\circ} \mathrm{C}$ a una velocidad de calentamiento de $10^{\circ} \mathrm{C} / \mathrm{min}$. A partir de las curvas de calentamiento, en presencia del flujo de aire, se determinaron las temperaturas de inicio de oxidación. Los datos se analizaron utilizando el programa Universal Analysis 2000, versión 4.5 A (TA Instruments). Cada prueba se realizó tres veces.

\section{RESULTADOS Y DISCUSIÓN}

\section{Propiedades físicas y químicas}

Se determinaron las siguientes propiedades físicas y químicas de la OC: índice de acidez, índice de yodo, índice de saponificación y porcentaje de humedad (Cuadro I). De acuerdo con los parámetros para el biodiésel y según las normas de la ASTM se determinó que la viscosidad, el índice de acidez y la humedad en la oleorresina exceden los límites permitidos por la normativa. Entonces, para desarrollar la producción de biodiésel de OC es necesario reducir estos valores, con el propósito de garantizar el funcionamiento eficaz y seguro del motor. Dado el contenido de fósforo en la OC se descartó la etapa de desgomado.

CUADRO I. PROPIEDADES FÍSICAS Y QUÍMICAS DE LA OLEORRESINA DE COPAIBA CRUDA

\begin{tabular}{lcc}
\hline Propiedad & Unidades & Valor \\
\hline Densidad a $23{ }^{\circ} \mathrm{C}$ & $(\mathrm{g} / \mathrm{mL})$ & 0.966 \\
Viscosidad a $40{ }^{\circ} \mathrm{C}$ & $\left(\mathrm{mm}^{2} / \mathrm{s}\right)$ & 53.3 \\
Acidez & $(\% \mathrm{p} / \mathrm{p} \mathrm{A}$, oleico $)$ & 17.99 \\
Índice de yodo & $(\mathrm{g} \mathrm{yodo} / 100 \mathrm{~g})$ & 189.1 \\
Índice de saponificación & $(\mathrm{mg} \mathrm{KOH} / \mathrm{g})$ & 48.6 \\
Contenido de fósforo & $(\mathrm{ppm})$ & $<0.1$ \\
Contenido de humedad & $(\%)$ & 1.7 \\
\hline
\end{tabular}

En el cuadro II se muestra el perfil de ácidos grasos de la OC. Se aprecia que los ácidos en mayor proporción son el oleico (14.72\%), linoleico $(7.43 \%)$ y el palmítico $(4.45 \%)$. En general, el $30 \%$ de la OC corresponde a ácidos grasos. El porcentaje restante corresponde a la fracción de sesquiterpenos y diterpenos presentes en la oleorresina.

En la literatura se ha reportado la presencia de ácidos resínicos para la especie Copaifera reticulata, como el kaurenoico (3.6\%) y el copálico (2.4\%), este último considerado como el diterpeno característico del género Copaifera; asimismo, de hidrocarburos sesquiterpénicos 
CUADRO II. PERFIL DE ÁCIDOS GRASOS DE LA OLEORRESINA DE Copaifera reticulata

\begin{tabular}{llcllr}
\hline Ácido graso & & $\begin{array}{c}\text { Resultado } \\
(\mathrm{g} / 100 \mathrm{~g})\end{array}$ & Ácido graso & $\begin{array}{c}\text { Resultado } \\
(\mathrm{g} / 100 \mathrm{~g})\end{array}$ \\
\hline Ácido hexanoico & $\mathrm{C} 6: 0$ & 0.53 & Ácido cis-9 oleico & $\mathrm{C} 18: 1$ & 14.72 \\
Ácido caprílico & $\mathrm{C} 8: 0$ & 0.03 & Ácido linolelaidico & $\mathrm{C} 18: 2 \mathrm{t}$ & 0.07 \\
Ácido caproico & $\mathrm{C} 10: 0$ & 1.52 & Ácido linoleico & $\mathrm{C} 18: 2$ & 7.43 \\
Ácido mirístico & $\mathrm{C} 14: 0$ & 0.23 & Ácido araquídico & $\mathrm{C} 20: 0$ & 0.08 \\
Ácido pentadecanoico & $\mathrm{C} 15: 0$ & 0.11 & Ácido linolénico & $\mathrm{C} 18: 3$ & 0.06 \\
Ácido cis-10 pentadecanoico & $\mathrm{C} 15: 0$ & 0.64 & Ácido cis 11-14 eicosadienoico & $\mathrm{C} 20: 2$ & 0.08 \\
Ácido palmítico & $\mathrm{C} 16: 0$ & 4.45 & Ácido erúcico & $\mathrm{C} 22: 1$ & 0.06 \\
Ácido palmitoleico & $\mathrm{C} 16: 1$ & 0.08 & Ácido nervónico & $\mathrm{C} 24: 1$ & 2.24 \\
Acido esteárico & $\mathrm{C} 18: 0$ & 0.8 & & & \\
\hline
\end{tabular}

como el $\beta$-cariofileno, bergamoteno y $\alpha$-copaeno en $40.9 \%, 4.1 \%$ y $3.0 \%$, respectivamente, como principales constituyentes de la oleorresina (Santos et al. 2008). Sin embargo, este resultado difiere de otras investigaciones, en las que se afirma que el bisaboleno y el $\alpha$-bergamoteno son los principales constituyentes de la oleorresina de Copaifera reticulata (HerreroJáuregui et al. 2011, Leandro et al. 2012, Bardají et al. 2016). Como se mencionó, factores ambientales como luminosidad, calidad del suelo, estacionalidad, así como la infección por hongos y diversos patógenos, pueden afectar la composición química de la oleorresina y hacer aún más compleja la determinación de sesquiterpenos y ácidos resínicos constituyentes (Herrero-Jáuregui et al. 2011, Leandro et al. 2012).

\section{Neutralización}

Con el método empleado para el refinado de la OC se logró reducir en un $97 \%$ la acidez de la oleorresina después del tratamiento con $\mathrm{NaOH}$. Sin embargo, este método dio lugar a la formación de jabones, con lo cual se redujo la cantidad de aceite disponible para la transesterificación. La neutralización por vía química junto con los procesos de desecado y filtrado redujeron en $55.3 \%$ la masa inicial de oleorresina. Se presentó una reducción del $5.7 \%$ y $63 \%$ en los valores de densidad y viscosidad, respectivamente. La OC refinada tiene una densidad de $0.911 \mathrm{~g} / \mathrm{mL}$ y una viscosidad cinemática de $19.8 \mathrm{~mm}^{2} / \mathrm{s}$. Estos resultados demuestran que a partir del proceso de refinado es posible reducir la viscosidad de la oleorresina, con miras a aplicarla como biocombustible.

\section{Transesterificación}

Luego de la transesterificación, la viscosidad de la muestra $\left(8.5 \mathrm{~mm}^{2} / \mathrm{s}\right)$ fue superior en comparación con el diésel de petróleo y el biodiésel según las normas ASTM, característica determinada por la estructura química de los metil ésteres de ácidos grasos producidos, en particular el grado de instauración y largo de cadena carbonada.

El uso de $\mathrm{NaOH}$ en el refinado y como catalizador en el proceso de transesterificación, puede aumentar la viscosidad del producto. Es preciso aclarar que, pese a compensar los niveles de acidez en la oleorresina, la neutralización de los ácidos grasos libres y la hidrólisis de los triglicéridos y posterior neutralización generan jabones inactivos catalíticamente que afectan la viscosidad del aceite (Narváez-Rincón et al. 2004). Aunque se esperaba reducir en mayor proporción la viscosidad del biocombustible para garantizar el cumplimento de la norma, la formación de jabones no lo permitió.

Si bien el uso de este biocombustible favorecería la lubricidad de las piezas en el motor como resultado de una viscosidad ligeramente superior al patrón estándar, podría generar complicaciones en el funcionamiento de los inyectores de la máquina, en particular a bajas temperaturas. En tal caso, es posible que un incremento en la viscosidad afecte la fluidez del combustible, por lo que no se aconseja su uso directo como combustible (Demirbas 2008, Atabani et al. 2012). Para asegurar una inyección adecuada del combustible en el motor y mantener un funcionamiento adecuado de los inyectores, se podría considerar el uso del biocombustible obtenido en mezclas con gasóleo.

El rendimiento global para la producción de biodiésel a partir de la OC cruda, mediante el método aplicado fue del $10 \%$ aproximadamente. Se aclara que los procesos de refinado y transesterificación del aceite generaron pérdidas en la materia prima disponible del 55.3 y $89.8 \%$, respectivamente (Cuadro III). La inducción de reacciones de saponificación durante la transesterificación afectó la eficiencia del $\mathrm{NaOH}$ como catalizador, así como 
CUADRO III. CANTIDADES DE MATERIA PRIMA EN CADA UNA DE LAS ETAPAS DEL PROCESO DE PRODUCCIÓN DE BIODIÉSEL DE COPAIBA

\begin{tabular}{lcccc}
\hline Etapa & $\begin{array}{c}\text { Masa } \\
\text { inicial }(\mathrm{g})\end{array}$ & $\begin{array}{c}\text { Masa } \\
\text { final }(\mathrm{g})\end{array}$ & $\begin{array}{c}\text { \% pérdida } \\
\text { de masa* }\end{array}$ & $\begin{array}{c}\text { \% rendimiento } \\
\text { reacción }\end{array}$ \\
\hline Secado-filtrado & 3000 & 2880 & 4 & - \\
Neutralización & 2880 & 1341 & 55.3 & 46.6 \\
Transesterificación & 1341 & 307 & 89.8 & 22.9 \\
\cline { 2 - 5 } & \multicolumn{2}{c}{$\%$ de Rendimiento global* } & 10.2 \\
\hline
\end{tabular}

*Respecto de la masa inicial de oleorresina

el rendimiento en la producción de metil ésteres y dificultó los procesos de separación y purificación del biodiésel de copaiba (Encinar et al. 2005, TaciasPascacio et al. 2016).

De acuerdo con los resultados obtenidos, la ruta escogida para modificar las propiedades físicas y químicas de la OC y ajustar estos parámetros a las propiedades exigibles para biocombustibles, no es apta. Se requieren estudios posteriores para establecer procesos que permitan aprovechar el potencial de la materia prima y así obtener rendimientos superiores en cuanto a la producción de biodiésel a partir de OC.

\section{Estudio FTIR}

La caracterización estructural de los diversos grupos funcionales presentes en la OC se realizó mediante espectroscopia infrarroja. En el espectro de FTIR, mostrado en la figura 2, se aprecian 17 grandes bandas de absorción en una secuencia uniforme, de modo que los espectros para la oleorresina cruda y la oleorresina filtrada y secada sólo difieren en el nivel de absorbancia de la banda correspondiente a los enlaces -OH. El uso de $\mathrm{Na}_{2} \mathrm{SO}_{4}$ anhidro para desecar la muestra permitió reducir en aproximadamente $53 \%$ el contenido de humedad en la oleorresina.

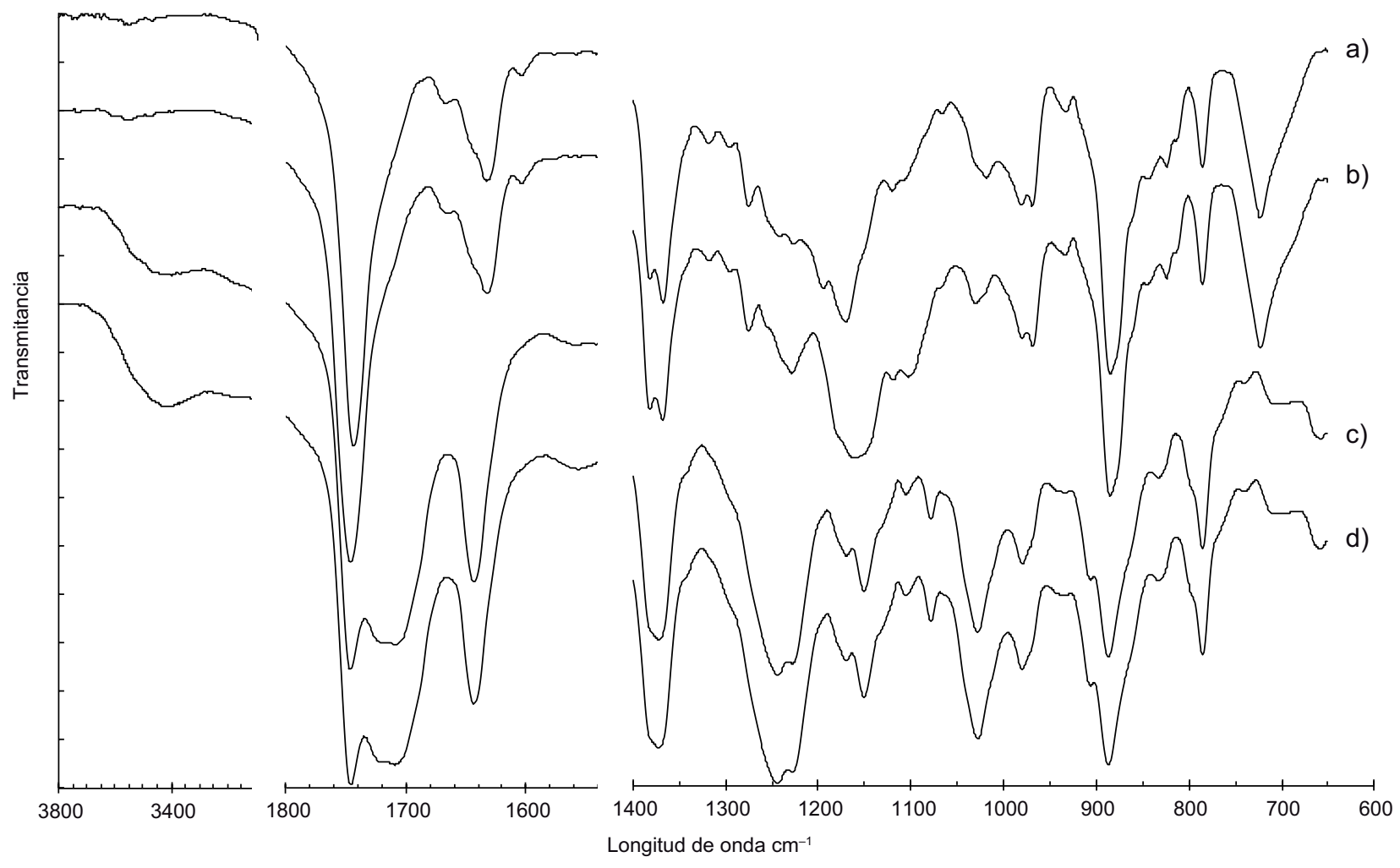

Fig. 2. Espectroscopia infrarroja por transformada de Fourier para la oleorresina de copaiba (OC) cruda y modificada, y el biodiésel de copaiba: a) biodiésel de copaiba; b) OC refinada; c) OC secada y filtrada, y d) OC cruda 
En principio, en el espectro infrarrojo se observa para la OC cruda una señal correspondiente a la tensión $-0 \mathrm{H}$ en $3399 \mathrm{~cm}^{-1}$ La banda centrada en $3077 \mathrm{~cm}^{-1}$ se asocia con la vibración del estiramiento $=\mathrm{C}-\mathrm{H}$ de dobles enlaces olefínicos. Los picos en 2926 y $2869 \mathrm{~cm}^{-1}$ se relacionan con vibraciones asimétricas y simétricas de extensión -C-H del grupo metileno, respectivamente. La banda centrada en $1746 \mathrm{~cm}^{1}$ refleja la absorbancia del grupo carbonilo $-\mathrm{C}=0$, característica del grupo éster en los triglicéridos (Oriani et al. 2016).

La señal originada en $1709 \mathrm{~cm}^{1}$ se atribuye al estiramiento de $\mathrm{C}=\mathrm{O}$ en los ácidos carboxílicos (Marquele-Oliveira et al. 2016). El pico en $1643 \mathrm{~cm}^{-1}$ se asocia con la vibración de la deformación $\mathrm{C}=\mathrm{C}$ de los dobles enlaces olefínicos. La tensión en $1557 \mathrm{~cm}^{-1}$ es atribuible a la vibración del anillo aromático (Bruni y Guglielmi 2014). Las bandas originadas en 1443 y $1372 \mathrm{~cm}^{-1}$ se asignan a la vibración de la flexión -C-H de los grupos $\mathrm{CH}_{2}$ y $\mathrm{CH}_{3}$ de los hidrocarburos alifáticos. Las bandas 1244, 1170, 1150 y $1105 \mathrm{~cm}^{-1}$ se asocian con las vibraciones del estiramiento del grupo -C-O en ácidos carboxílicos y C-O en ésteres, respectivamente. El pico en $1026 \mathrm{~cm}^{-1}$ se puede atribuir a la vibración de los enlaces -C-H en compuestos aromáticos. Finalmente, las bandas entre 980 y 785 $\mathrm{cm}^{-1}$ se explican por la vibración de la flexión fuera del plano de las olefinas.

Los espectros infrarrojos, tanto para la oleorresina modificada como para el biodiésel obtenido, difieren de la OC cruda únicamente en la intensidad de las bandas de absorción (Fig. 2). Se observa la definición de las bandas correspondientes a la vibración de compuestos del grupo carbonilo, específicamente los ésteres, así como la disminución de las bandas de absorción de los ácidos carboxílicos en cuanto resultado de la transesterificación. Adicionalmente, se evidencia la disminución en la absorbancia de compuestos aromáticos, eliminados casi en su totalidad durante las etapas de refinado y transesterificación.

\section{Comportamiento a bajas temperaturas}

Las curvas de enfriamiento-calentamiento para la $\mathrm{OC}$ cruda evidencian un comportamiento estable de la muestra entre -60 y $50{ }^{\circ} \mathrm{C}$, en la que no se aprecian procesos de cristalización o fusión (Fig. 3). Respecto de la oleorresina refinada, se aprecia cómo la modificación química dio lugar a cambios de fase, al promover reacciones exotérmicas y endotérmicas ante variaciones en el flujo de calor. Se puede observar cómo en la etapa de enfriamiento a $-44.53{ }^{\circ} \mathrm{C}$ se origina el primer cambio de fase o cristalización. Posteriormente, al aumentar su temperatura se presentan dos reacciones endotérmicas a -45.76 y $-26.52{ }^{\circ} \mathrm{C}$, en las que se funde la muestra.

En cuanto al biodiésel de copaiba, en la figura 3 y el cuadro IV se aprecia cómo los cambios de fase se relacionan con mayor cantidad de energía, tanto para la formación de cristales $\left({ }^{\circ} \Delta \mathrm{H}_{\mathrm{c}}\right)$ como para la fusión de la muestra $\left({ }^{\circ} \Delta H_{\mathrm{m}}\right)$, con relación a la oleorresina refinada. Se observa una exoterma en la etapa de enfriamiento que coincide con el

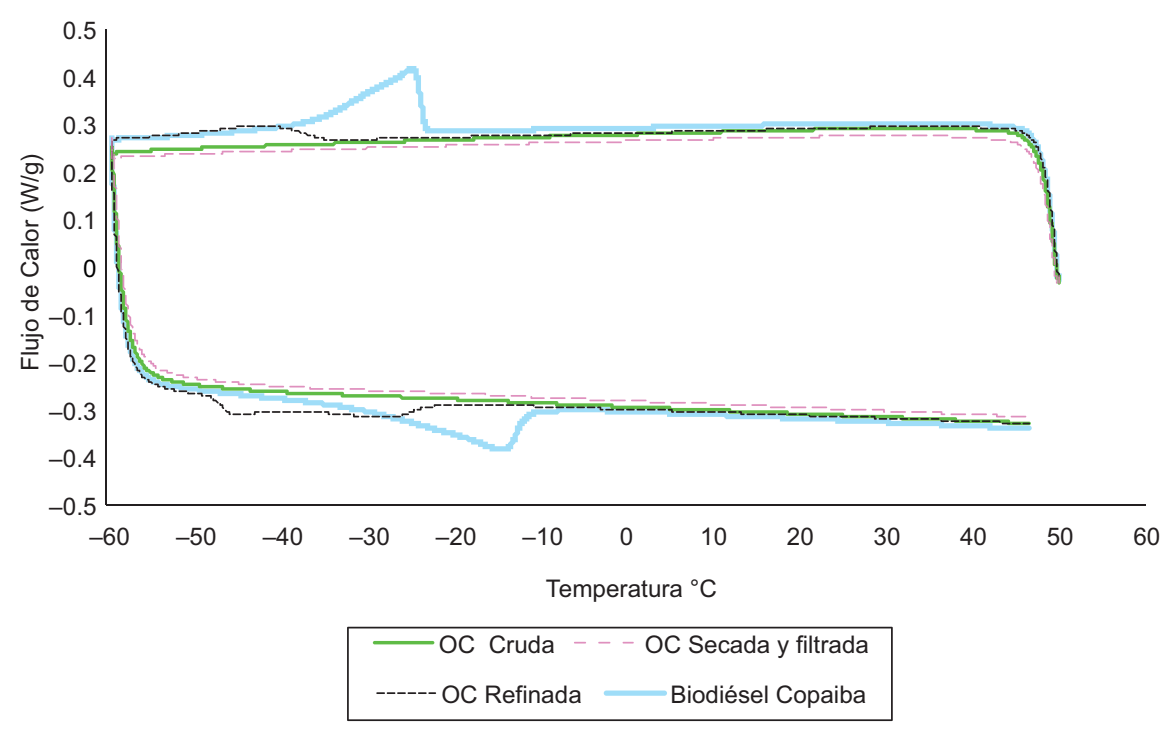

Fig. 3. Curvas de enfriamiento-calentamiento de la oleorresina de copaiba (OC) cruda, secada y filtrada, refinada, y el biodiésel de copaiba 
CUADRO IV. ENTALPÍAS DE CRISTALIZACIÓN Y FUSIÓN EN LA OLEORRESINA REFINADA Y EL BIODIÉSEL DE COPAIBA

\begin{tabular}{|c|c|c|c|c|c|c|c|c|c|c|}
\hline \multirow{2}{*}{$\begin{array}{l}\text { Pico } \\
\text { núm. }\end{array}$} & \multicolumn{5}{|c|}{ Oleorresina de copaiba refinada } & \multicolumn{5}{|c|}{ Biodiésel de copaiba } \\
\hline & $\mathrm{T}\left({ }^{\circ} \mathrm{C}\right)$ & ${ }^{\circ} \Delta \mathrm{H}_{\mathrm{c}}(\mathrm{J} / \mathrm{g})$ & $\% \mathrm{CV}$ & ${ }^{\circ} \Delta \mathrm{H}_{\mathrm{m}}(\mathrm{J} / \mathrm{g})$ & $\% \mathrm{CV}$ & $\mathrm{T}\left({ }^{\circ} \mathrm{C}\right)$ & ${ }^{\circ} \Delta H_{c}(J / g)$ & $\% \mathrm{CV}$ & ${ }^{\circ} \Delta \mathrm{H}_{\mathrm{m}}(\mathrm{J} / \mathrm{g})$ & $\% \mathrm{CV}$ \\
\hline 1 & -44.53 & 1.92 & 0.69 & - & - & - & 6.25 & 0.29 & - & - \\
\hline 2 & -45.76 & - & - & 0.59 & 0.21 & -24.68 & - & - & 5.44 & 1.19 \\
\hline 3 & -26.52 & - & - & 0.72 & 0.43 & -14.42 & - & - & - & - \\
\hline
\end{tabular}

${ }^{\circ} \Delta H_{\mathrm{c}}$ : energía asociada a la formación de cristales en la muestra, ${ }^{\circ} \Delta H_{\mathrm{m}}$ : energía asociada a la fusión de la muestra, $\mathrm{CV}$ : coeficiente de variación

inicio de la formación de cristales $\left(T_{\mathrm{n}}\right) \mathrm{a}-23.23^{\circ} \mathrm{C}$ con cristalización a $-24.68^{\circ} \mathrm{C}$. Posteriormente, se aprecia una endoterma durante la etapa de calentamiento a $-14.42{ }^{\circ} \mathrm{C}$ que se relaciona con la fusión de la muestra. Los cambios entre los procesos de cristalización y fusión son un fenómeno conocido en ácidos grasos insaturados y ésteres alquílicos (Issariyakul et al. 2008, Yilmaz et al. 2015).

En el cuadro IV se presentan los calores latentes asociados a puntos de cristalización y fusión para la oleorresina refinada y el biodiésel de copaiba, con sus respectivos coeficientes de variación $(\mathrm{CV})$.

\section{Estabilidad oxidativa}

La estabilidad oxidativa de la OC cruda y refinada, y el biodiésel obtenido a partir de su transesterificación fue estimada por DSC. Los termogramas correspondientes se presentan en la figura 4 , en la que se aprecia que los tratamientos previos a los que fue sometida la oleorresina para reducir los niveles de acidez y viscosidad del aceite dan lugar a variaciones importantes en su comportamiento a temperaturas altas. La OC cruda presenta un par de picos endotérmicos en 161 y $173{ }^{\circ} \mathrm{C}$ que pueden atribuirse a la volatilización de compuestos de bajo peso molecular y posterior oxidación de la muestra.

Claramente se aprecia que los compuestos volátiles presentes en la oleorresina cruda son eliminados casi en su totalidad después de los procesos de refinado y transesterificación, resultado acorde con los perfiles de espectroscopia infrarroja en cada caso (Fig. 2). Tanto la OC refinada como el biodiésel de copaiba presentan puntos de inflexión similares, por encima y por debajo de la temperatura de inicio de oxidación de la oleorresina en su estado virgen, que pueden atribuirse a la oxidación parcial de los compuestos de cada una de las muestras (Fig. 4 y Cuadro V).

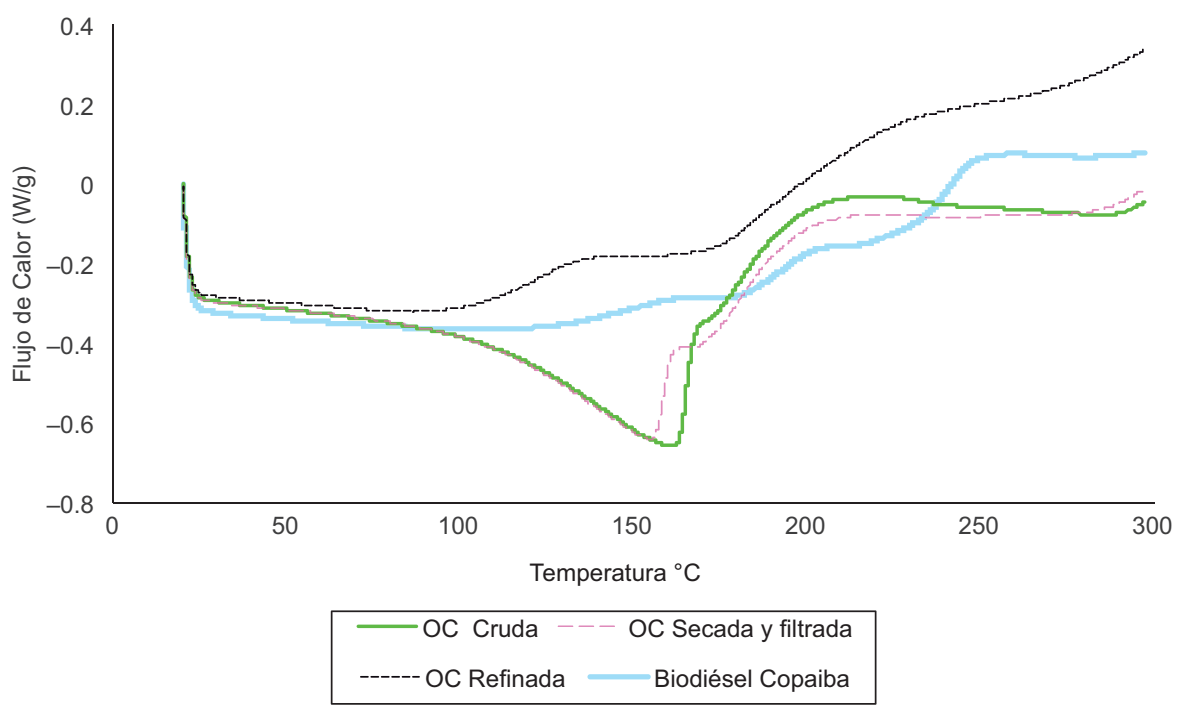

Fig. 4. Curvas de estabilidad oxidativa de la oleorresina de copaiba (OC) cruda, secada y filtrada, y refinada, así como del biodiésel de copaiba 
CUADRO V. TEMPERATURAS DE OXIDACIÓN PARA LA OLEORRESINA DE COPAIBA (OC) CRUDA, SECADA Y FILTRADA, Y REFINADA, ASÍ COMO DEL BIODIÉSEL DE COPAIBA

\begin{tabular}{lcccc}
\hline \multirow{2}{*}{$\begin{array}{l}\text { Pico } \\
\text { núm. }\end{array}$} & $\begin{array}{c}\text { OC } \\
\text { cruda }\end{array}$ & $\begin{array}{c}\text { OC secada } \\
\text { y filtrada }\end{array}$ & $\begin{array}{c}\text { OC } \\
\text { refinada }\end{array}$ & $\begin{array}{c}\text { Biodiésel } \\
\text { de copaiba }\end{array}$ \\
\cline { 2 - 5 } & \multicolumn{4}{c}{ Temperatura $\left({ }^{\circ} \mathrm{C}\right)$} \\
\hline 1 & 161.39 & 155.36 & 169.93 & 128.05 \\
2 & 173.69 & 169.93 & 174.56 & 180.92 \\
3 & - & - & - & 220.56 \\
\hline
\end{tabular}

Los mecanismos de degradación de la oleorresina pueden incluir procesos de oxidación, polimerización e isomerización y reacciones de escisión en ácidos grasos, así como procesos de volatilización de sesquiterpenos, que afectan tanto su composición química, cualitativa y cuantitativa, como su respuesta ante cambios de temperatura en presencia de $\mathrm{O}_{2}$.

Como se aprecia en el cuadro $\mathbf{V}$, al considerar la descomposición total de la muestra, se puede observar que la estabilidad oxidativa del biodiésel de copaiba es mayor y se incrementa en 46 y $47^{\circ} \mathrm{C}$ aproximadamente, en relación con la oleorresina cruda y la refinada, respectivamente (Scalarone et al. 2002, Cartoni et al. 2004, Fox y Stachowiak 2007). De acuerdo con los resultados obtenidos, los procesos de refinado y transesterificación favorecen la estabilidad del biocombustible ante procesos de degradación en ambiente oxidante.

\section{Capacidad calorífica}

La capacidad calorífica $(\mathrm{Cp})$ de las muestras se determinó por el método calorimétrico según la norma ASTM E1269 [ASTM 2005b]. En la figura 5 se observa la variación en el valor del Cp de las muestras en el intervalo de las temperaturas de estudio (46.85$66.85^{\circ} \mathrm{C}$ ). Pese a que la tendencia es la misma y los cambios en el $\mathrm{Cp}$ son directamente proporcionales a los cambios en la temperatura, la oleorresina en su estado crudo presenta una capacidad calorífica superior con relación a su versión modificada y al biodiésel de copaiba. Se resalta que el refinado del aceite disminuye notablemente el Cp de la muestra, que luego de la reacción de alcoholis se incrementa nuevamente, pero presenta valores inferiores respecto de la OC cruda. Los valores de capacidad calorífica encontrados guardan relación con los valores de $\mathrm{Cp}$ para mezclas de metil ésteres de ácidos grasos (Pauly et al. 2014).

En el cuadro VI se presentan las características físicas y químicas de la OC cruda y refinada, y el biodiésel de copaiba. En todos los casos, el punto de inflamación determinado a partir del método de copa cerrada Pensky Martens es ligeramente inferior al límite que establece la norma para el biodiésel. Esto se puede atribuir a la presencia de compuestos volátiles en la oleorresina, que afectan esta propiedad. Sin embargo, los resultados experimentales para cada muestra son superiores a los valores permitidos para el punto de inflamación del combustible diésel, según la norma ASTM D975 (ASTM 2016b) (Atabani et al. 2013, Silitonga et al. 2013).

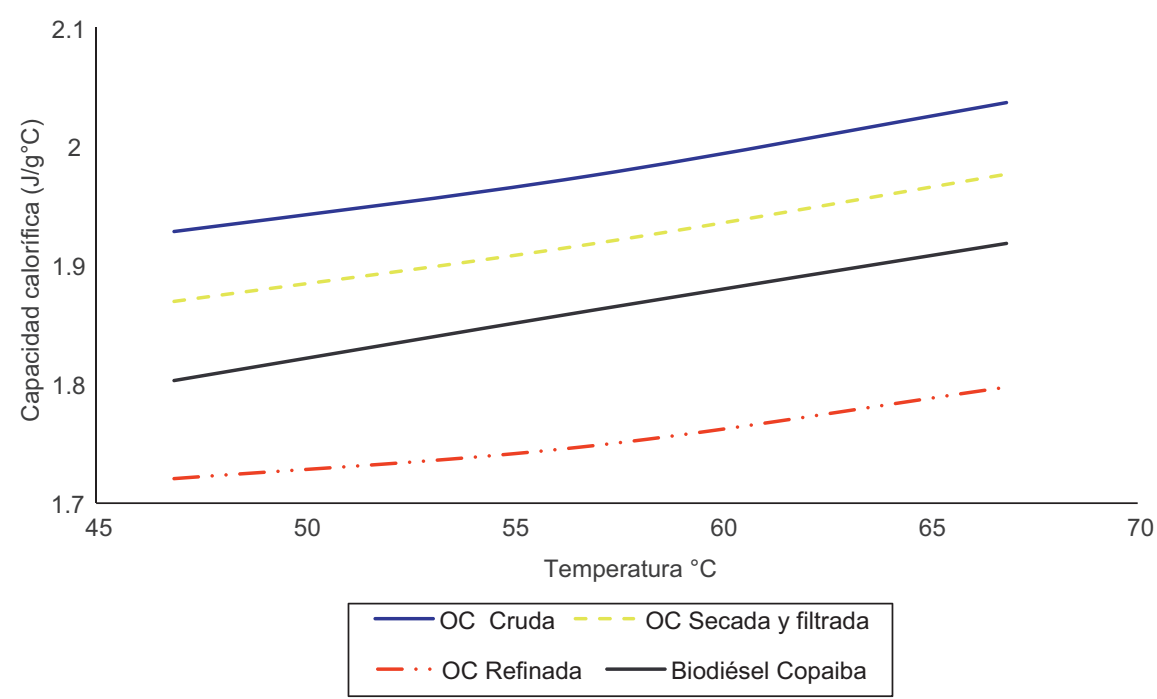

Fig. 5. Capacidades caloríficas de la oleorresina de copaiba (OC) cruda, secada y filtrada, y refinada, así como del biodiésel de copaiba 
CUADRO VI. COMPARATIVO DE LAS PROPIEDADES FÍSICAS Y QUÍMICAS DE LA OLEORRESINA DE COPAIBA (OC) CRUDA, SECADA Y FILTRADA, Y REFINADA, ASÍ COMO DEL BIODIÉSEL DE COPAIBA

\begin{tabular}{lcccccc}
\hline Propiedad & $\begin{array}{c}\text { OC } \\
\text { cruda }\end{array}$ & $\begin{array}{c}\text { OC } \\
\text { secada y filtrada }\end{array}$ & $\begin{array}{c}\text { OC } \\
\text { refinada }\end{array}$ & $\begin{array}{c}\text { Biodiésel } \\
\text { copaiba }\end{array}$ & $\begin{array}{c}\text { ASTM } \\
\text { D6751* } \\
\text { (ASTM 2015b) }\end{array}$ & $\begin{array}{c}\text { ASTM } \\
\text { D975** } \\
\text { (ASTM 2016b) }\end{array}$ \\
\hline Densidad $(\mathrm{g} / \mathrm{ml})$ & 0.966 & 0.965 & 0.911 & 0.901 & 0.880 & 0.850 \\
Viscosidad $40{ }^{\circ} \mathrm{C}\left(\mathrm{mm}^{2} / \mathrm{s}\right)$ & 53.3 & 50.1 & 19.8 & 8.5 & $1.9-6.0$ & $1.9-4.1$ \\
Índice de acidez $(\mathrm{mg} / \mathrm{g} \mathrm{KOH})$ & 17.99 & - & 0.57 & - & 0.5 máx, & - \\
Punto de inflamación $\left({ }^{\circ} \mathrm{C}\right)$ & 98 & 99 & 98 & 96 & $100-170$ & $60-80$ \\
Punto de fluidez $\left({ }^{\circ} \mathrm{C}\right)$ & -24 & -24 & $<-39$ & $<-39$ & $-15-16$ & -35 \\
\hline
\end{tabular}

* Especificación estándar para biocombustibles; ** especificación estándar para aceites combustibles diésel; ASTM: Sociedad Estadounidense para Pruebas y Materiales

Las temperaturas más bajas en las que la $\mathrm{OC}$ cruda, modificada y el biodiésel de copaiba pueden fluir superan en todos los casos los límites establecidos por la norma ASTM D97 (ASTM 2005c) para biocombustibles. En cuanto a la OC refinada y el biodiésel de copaiba, también sobrepasan el límite para combustibles de origen fósil, con un valor inferior a $-39^{\circ} \mathrm{C}$ (Atabani et al. 2012, Silitonga et al. 2013).

De acuerdo con el comportamiento exhibido por las muestras y en particular el biodiésel de copaiba en condiciones extremas de temperatura, estos valores evitarían la obstrucción de los filtros y conductos del combustible, así como problemas de funcionamiento del motor, ya sea por falta de combustible, fallas de partida o lubricación inadecuada a bajas temperaturas.

\section{CONCLUSIONES}

Se concluye que los métodos empleados en esta investigación para la modificación química de la oleorresina no son eficientes, ya que se genera la pérdida de gran parte de la materia prima disponible. Sin embargo, las propiedades físicas y químicas medidas en la oleorresina cruda, modificada y el biocombustible obtenido, permiten establecer el potencial de este recurso para la formulación de biodiésel.

En todos los casos, el comportamiento a baja temperatura podría traducirse como una contribución significativa a la reducción de problemas de fluidez de combustible en condiciones extremas de temperatura. Sin embargo, propiedades como la viscosidad y la densidad, tanto de la oleorresina como del biodiésel de copaiba, condicionan su uso directo como combustible, pues debido a su alta viscosidad podría generar obstrucción tanto en filtros como en inyectores del motor. Los puntos de inflamación se encuentran por debajo del valor estándar para biocombustibles, pero superan los límites para combustibles de origen fósil, por lo que estarían en condiciones seguras de almacenamiento y transporte.

De acuerdo con los resultados obtenidos, los procesos de refinado y transesterificación influyeron positivamente en la estabilidad oxidativa de la oleorresina de copaiba cruda. Evidentemente, la estabilidad de la oleorresina refinada y el biodiésel de copaiba fue superior en 46 y $47^{\circ} \mathrm{C}$, respectivamente.

En general, la oleorresina de copaiba constituye una alternativa ecosostenible que se podría emplear para mejorar las propiedades de flujo en frío en combustibles, tanto en su forma cruda como modificada o transesterificada. Su uso, aunque condicionado de forma directa, podría considerarse en mezclas con gasóleo y otros biocombustibles, a fin de mejorar las propiedades a bajas temperaturas. En estudios posteriores sería posible establecer otro proceso eficiente para la modificación química de la oleorresina.

\section{AGRADECIMIENTOS}

Este estudio forma parte del proyecto de investigación INV-ING-2122 financiado por la Vicerrectoría de Investigaciones de la Universidad Militar Nueva Granada, vigencia 2016.

\section{REFERENCIAS}

Agarwal A.K. (2007). Biofuels (alcohols and biodiesel) applications as fuels for internal combustion engines. Prog. Energy Combust. Sci. 33 (3), 233-271.

DOI: $10.1016 /$ j.pecs.2006.08.003

ASTM (2005a). Standard D92. Standard test method for flash and fire points by Cleveland open cup tester. American Society for Testing and Materials. 
ASTM (2005b). Standard E1269. Standard test method for determining specific heat capacity by differential scanning calorimetry. American Society for Testing and Materials.

ASTM (2005c). Standard D97. Standard test method for pour point of petroleum products. American Society for Testing and Materials.

ASTM (2015a). Standard D445. Standard test method for kinematic viscosity of transparent and opaque liquids (and calculation of dynamic viscosity). American Society for Testing and Materials.

ASTM (2015b). Standard D6751. Standard specification for biodiesel fuel blend stock (B100) for middle distillate fuels. American Society for Testing and Materials.

ASTM (2016a). Standard E203. Standard test method for water using volumetric Karl Fischer titration. American Society for Testing and Materials.

ASTM (2016b). Standard D975. Standard specification for diesel fuel oils. American Society for Testing and Materials.

Atabani A.E., Silitonga A.S., Badruddin I.A., Mahlia T.M.I., Masjuki H.H. y Mekhilef S. (2012). A comprehensive review on biodiesel as an alternative energy resource and its characteristics. Renew. Sustain. Energy Rev. 16 (4), 2070-2093.

DOI: $10.1016 /$ j.rser.2012.01.003

Atabani A.E., Silitonga A.S., Ong H.C., Mahlia T.M.I., Masjuki H.H., Badruddin I.A. y Fayaz H. (2013). Non-edible vegetable oils: A critical evaluation of oil extraction, fatty acid compositions, biodiesel production, characteristics, engine performance and emissions production. Renew. Sustain. Energy Rev. 18, 211-245. DOI: 10.1016/j.rser.2012.10.013

Bardají D.K.R., Da Silva J.J.M., Bianchi T.C., De Souza Eugênio D., De Oliveira P.F., Leandro L.F., Rogez H.L.G., Venezianni R.C.S., Ambrosio S.R., Tavares D.C., Bastos J.K. y Martins C.H.G. (2016). Copaifera reticulata oleoresin: Chemical characterization and antibacterial properties against oral pathogens. Anaerobe 40, 18-27.

DOI: 10.1016/j.anaerobe.2016.04.017

Bruni S. y Guglielmi V. (2014). Identification of archaeological triterpenic resins by the non-separative techniques FTIR and 13C NMR: The case of Pistacia resin (mastic) in comparison with frankincense. Spectrochim. Acta. A. Mol. Biomol. Spectrosc. 121, 613-622.

DOI: $10.1016 /$ j.saa.2013.10.098

Calvin M. (1980). Hydrocarbons from plants: Analytical methods and observations. Naturwissenschaften 67, 525-533. DOI: 10.1007/BF00450661

Calvin M. (1983). New sources for fuel and materials. Science 219, 24-26. DOI: 10.1126/science.219.4580.24
Calvin M. (1987). High-energy fuels and materials from plants. J. Chem. Educ. 64 (4), 335.

DOI: $10.1021 / \mathrm{ed} 064 \mathrm{p} 335$

Cartoni G., Russo M.V., Spinelli F. y Talarico F. (2004). GC-MS characterisation and identification of natural terpenic resins employed in works of art. Ann. Chim. 94 (11), 767-782.

DOI: $10.1002 /$ adic. 200490098

CEPAL (2007). Producción de biomasa para biocombustibles líquidos: el potencial de América Latina y el Caribe. Comisión Económica para América Latina y el Caribe. Santiago de Chile, 84 pp.

Chen F., al-Ahmad H., Joyce B., Zhao N., Köllner T.G., Degenhardt J. y Stewart C.N. (2009). Within-plant distribution and emission of sesquiterpenes from Copaifera officinalis. Plant Physiol. Biochem. 47 (11-12), 1017-1023.

DOI: 10.1016/j.plaphy.2009.07.005

Da Silva Medeiros R. y Vieira G. (2008). Sustainability of extraction and production of copaiba (Copaifera multijuga Hayne) oleoresin in Manaus, AM, Brazil. For. Ecol. Manag. 256 (3), 282-288.

DOI: $10.1016 / \mathrm{j}$.foreco.2008.04.041

Delgado A.E., Aperador-Chaparro W. y González-Silva J.R. (2011). Influence of castor oil mix composition on the production of biofuel. Rev. Fac. Ing. Univ. Antioquia 48, 46-52.

Demirbas A. (2007). Importance of biodiesel as transportation fuel. Energ. Policy 35 (9), 4661-4670.

DOI: $10.1016 /$ j.enpol.2007.04.003

Demirbas A. (2008). Relationships derived from physical properties of vegetable oil and biodiesel fuels. Fuel 87 (8-9), 1743-1748.

DOI: 10.1016/j.fuel.2007.08.007

Encinar J.M., González J.F. y Rodríguez-Reinares A. (2005). Biodiesel from used frying oil. Variables affecting the yields and characteristics of the biodiesel. Ind. Eng. Chem. Res. 44 (15), 5491-5499.

DOI: $10.1021 / \mathrm{ie} 040214 \mathrm{f}$

ESA (2012). Ethiopian Standard ES ISO 10540-1. Animal vegetables fats and oils. Determination of phosphorus content. Part 1: Colorimetric method. Ethiopian Standards Agency.

Fox N.J. y Stachowiak G.W. (2007). Vegetable oil-based lubricants - A review of oxidation. Tribol. Int. 40 (7), 1035-1046.

DOI: $10.1016 /$ j.triboint.2006.10.001

Gaurav N., Sivasankari S., Kiran G., Ninawe A. y Selvin J. (2017). Utilization of bioresources for sustainable biofuels: A review. Renew. Sustain. Energy Rev. 73, 205-214. DOI: 10.1016/j.rser.2017.01.070

Gelmini F., Beretta G., Anselmi C., Centini M., Magni P., Ruscica M., Cavalchini A. y Maffei-Facino R. (2013). 
GC-MS profiling of the phytochemical constituents of the oleoresin from Copaifera langsdorffii Desf. and a preliminary in vivo evaluation of its antipsoriatic effect. Int. J. Pharm. 440 (2), 170-178.

DOI: 10.1016/j.ijpharm.2012.08.021

Gonçalves E.S., Silva J.R., Gomes C.L., Nery M.B.L., Navarro D.M.A.F., Santos, G.K.N., Silva-Neto J.C., Costa-Silva J.H., Araújo A.V. y Wanderley A.G. (2014). Effects of the oral treatment with Copaifera multijuga oil on reproductive performance of male Wistar rats. Rev. Bras. Farmacogn. 24 (3), 355-362. DOI: 10.1016/j.bjp.2014.07.014

Habibullah M., Masjuki H.H., Kalam M.A., Rahman S.M.A., Mofijur M., Mobarak H.M. y Ashraful A.M. (2015). Potential of biodiesel as a renewable energy source in Bangladesh. Renew. Sustain. Energ. Rev. 50, 819-834. DOI: 10.1016/j.rser.2015.04.149

Herrero-Jáuregui C., Casado M.A., Bichara-Zoghbi MG. y Martins-Da Silva RC. (2011). Chemical variability of Copaifera reticulata Ducke oleoresin. Chem. Biodivers. 8 (4), 674-685. DOI: 10.1002/cbdv.201000258

ICONTEC (1999). Norma Técnica Colombiana NTC 218 (2). Grasas y aceites vegetales y animales. Determinación del índice de acidez y de la acidez. Instituto Colombiano de Normas Técnicas y Certificación.

Issariyakul T., Kulkarni M.G., Meher L.C., Dalai A.K. y Bakhshi N.N. (2008). Biodiesel production from mixtures of canola oil and used cooking oil. Chem. Eng. J. 140 (1-3), 77-85. DOI: 10.1016/j.cej.2007.09.008

Klauberg C., Vidal E., Rodríguez L.C.E. y Díaz-Balteiro L. (2014). Determining the optimal harvest cycle for copaíba (Copaifera spp.) oleoresin production. Agric. Syst. 131, 116-122. DOI: 10.1016/j.agsy.2014.07.007

Kuss V.V., Kuss A.V., Rosa R.G., Aranda D.A.G. y Cruz Y.R. (2015). Potential of biodiesel production from palm oil at Brazilian Amazon. Renew. Sustain. Energy Rev. 50, 1013-1020. DOI: 10.1016/j.rser.2015.05.055

Leandro L.M., De-Sousa-Vargas F., Barbosa-Souza P.C., Neves-Oliveira J.K., Da Silva J.A. y Da Veiga-Junior V.F. (2012). Chemistry and biological activities of terpenoids from copaiba (Copaifera spp.) oleoresins. Molecules 17 (4), 3866-3889.

DOI: $10.3390 /$ molecules 17043866

Malins K., Kampars V., Kampare R., Prilucka J., Brinks J., Murnieks R. y Apseniece L. (2014). Properties of rapeseed oil fatty acid alkyl esters derived from different alcohols. Fuel 137, 28-35.

DOI: 10.1016/j.fuel.2014.07.091

Marquele-Oliveira F., Torres E.C., Da Silva-Barud H., Zoccal K.F., Faccioli L.H., Hori J.I. y Berretta A.A. (2016). Physicochemical characterization by AFM, FT-IR and DSC and biological assays of a promising antileishmania delivery system loaded with a natural
Brazilian product. J. Pharm. Biomed. Anal. 123, 195204. DOI: 10.1016/j.jpba.2016.01.045

Mata T.M., Martins A.A. y Caetano N.S. (2010). Microalgae for biodiesel production and other applications: A review. Renew. Sustain. Energ. Rev. 14 (1), 217-232. DOI: $10.1016 /$ j.rser.2009.07.020

Morone P. y Cottoni L. (2016). Biofuels: Technology, economics, and policy issues. En: Handbook of biofuels production, 2a ed. (R. Luque, C. Lin, K. Wilson, J. Clark, Eds.). Woodhead Publishing, Nueva Delhi, India, pp. 61-83.

Oriani V.B., Alvim I.D., Consoli L., Molina G., Pastore G.M. y Hubinger M.D. (2016). Solid lipid microparticles produced by spray chilling technique to deliver ginger oleoresin: Structure and compound retention. Food Res. Int. 80, 41-49.

DOI: 10.1016/j.foodres.2015.12.015

Pauly J., Kouakou A.C., Habrioux M. y Le Mapihan K. (2014). Heat capacity measurements of pure fatty acid methyl esters and biodiesels from 250 to $390 \mathrm{~K}$. Fuel 137, 21-27. DOI: 10.1016/j.fuel.2014.07.037

Pieri F.A., Mussi M.C. y Moreira M.A.S. (2009). Copaiba oil (Copaifera sp.): history, extraction, industrial applications and medicinal properties. Rev. Bras. Plantas Med. 11 (4), 465-472.

DOI: $10.1590 / \mathrm{S} 1516-05722009000400016$

Rashid U. y Anwar F. (2008). Production of biodiesel through optimized alkaline-catalyzed transesterification of rapeseed oil. Fuel 87 (3), 265-273.

DOI: 10.1016/j.fuel.2007.05.003

Rathore V., Newalkar B.L. y Badoni R.P. (2016). Processing of vegetable oil for biofuel production through conventional and non-conventional routes. Energ. Sustain. Dev. 31, 24-49.

DOI: $10.1016 /$ j.esd.2015.11.003

Narváez-Rincón P.C., Sánchez F.J., Torres J.A. y Poncede-León L.F. (2004). Producción de ésteres metílicos de ácidos grasos: variables asociadas al proceso de transformación. Ingeniería e Investigación 24 (2), 41-50.

Rodrigues T.M., Teixeira S. de P. y Machado S.R. (2011). The oleoresin secretory system in seedlings and adult plants of copaíba (Copaifera langsdorffii Desf., Leguminosae-Caesalpinioideae). Flora-Morphol. Distrib. Funct. Ecol. Plants 206 (6), 585-594. DOI: $10.1016 /$ j.flora.2010.10.002

Santos A.O., Ueda-Nakamura T., Dias Filho B.P., VeigaJunior V.F., Pinto A.C. y Nakamura C.V. (2008). Effect of Brazilian copaiba oils on Leishmania amazonensis. J. Ethnopharmacol. 120 (2), 204-208. DOI: $10.1016 /$ j.jep.2008.08.007

Scalarone D., Lazzari M. y Chiantore O. (2002). Ageing behaviour and pyrolytic characterisation of diterpenic 
resins used as art materials: colophony and Venice turpentine. J. Anal. Appl. Pyrolysis 64 (2), 345-361. DOI: $10.1016 / \mathrm{S} 0165-2370(02) 00046-3$

Silitonga A.S., Ong H.C., Mahlia T.M.I., Masjuki H.H. y Chong W.T. (2013). Characterization and production of Ceiba pentandra biodiesel and its blends. Fuel 108, 855-858. DOI: 10.1016/j.fuel.2013.02.014

Silva W.G., Cortesi N. y Fusari P. (2010). Copaiba oleoresin: evaluation of the presence of polycyclic aromatic hydrocarbons (PAHs). Braz. J. Pharm. Sci. 46 (3), 597-602. DOI: 10.1590/S1984-82502010000300025

Sitepu I.R., Garay L.A., Sestric R., Levin D., Block D.E., German J.B. y Boundy-Mills K.L. (2014). Oleaginous yeasts for biodiesel: Current and future trends in biology and production. Biotechnol. Adv. 32 (7), 1336-1360. DOI: 10.1016/j.biotechadv.2014.08.003
Tacias-Pascacio V.G., Quintero A.R. y Sánchez B.T. (2016). Evaluación y caracterización de grasas y aceites residuales de cocina para la producción de biodiésel: un caso de estudio. Rev. Int. Contam. Ambie. 32 (3), 303-313. DOI: 10.20937/RICA.2016.32.03.05 Veiga-Junior V.F. y Pinto A.C. (2002). The Copaifera L. genus. Quím. Nova 25 (2), 273-286. DOI: $10.1590 / \mathrm{S} 0100-40422002000200016$

Yılmaz E., Sevgi Arsunar E., Aydeniz B. y Güneşer O. (2015). Cold pressed Capia pepperseed (Capsicum annuиm L.) oils: Composition, aroma, and sensory properties. Eur. J. Lipid Sci. Technol. 117 (7), 10161026. DOI: $10.1002 /$ ejlt.201400276 